\title{
Tea seed oil vs. olive oil: plasma antioxidant activity in humans
}

\author{
Nuchanart Suealek ${ }^{1}$, Thipaporn Tharavanij ${ }^{2}$, Panadda Rojpibulstit ${ }^{3}$
}

\begin{abstract}
${ }^{I}$ Division of Pharmacology, Department of Preclinical Science, Faculty of Medicine, Thammasat University, Pathum Thani, Thailand, ${ }^{2}$ Endocrinology Unit, Department of Medicine, Faculty of Medicine, Thammasat University, Pathum Thani, Thailand, ${ }^{3}$ Division of Biochemistry, Department of Preclinical Science, Faculty of Medicine, Thammasat University, Pathum Thani, Thailand
\end{abstract}

Olive oil (OO) is a well-known healthy cooking oil, rich in monounsaturated fatty acids (MUFAs), especially oleic acid. Tea seed oil (TSO) is a relatively new cooking oil with an equivalent MUFA profile as OO. Tea seed oil has similar beneficial effects on human lipid profiles; however, plasma antioxidant activity during consumption of TSO and OO in healthy volunteers needs to be further investigated.

The maintained intake, randomized, crossover study for 3 weeks, with a 3-week washout period, was designed to compare TSO and OO diets on plasma antioxidant activity in healthy volunteers. The First Study Period, 12 healthy men were randomly given TSO or OO, as cooking oil at $40 \mathrm{~g} /$ day. Before and after the 3 -week study period, their plasma samples were analyzed for malondialdehyde (MDA) levels to assess oxidative stress. Additionally, plasma antioxidant enzyme activities, such as glutathione peroxidase (GPx), superoxide dismutase (SOD), and catalase (CAT) were also examined. In the Second Study Period, the participants were crossovered with the same design as the First Study Period. Cooking temperature and food ingredients were identical in both TSO and OO periods. Comparative data were analyzed using repeated-measures ANOVA.

After 3 weeks of study diet, the TSO diet did not produce oxidative stress, much like the OO diet. Additionally, TSO diet did not demonstrate significant differences in all plasma enzyme activities when compared with the first day. The OO diet showed a transient decrease in SOD activity and tended to decrease the CAT activity. However, at the end of the study, there were no significant differences in all plasma antioxidant enzyme activities between TSO and OO diets. This is most likely due to the similar oil composition of TSO and OO, especially MUFA profile.

In conclusion, after 3 weeks of the consumption of TSO, as cooking oil at $40 \mathrm{~g} /$ day, the TSO diet exhibited a neutral effect on oxidative stress and plasma enzyme activities similar to the $\mathrm{OO}$ diet in healthy volunteers. Anyway, to better understand TSO's other antioxidant capacities, such as on high oxidative stress status, more research is needed. 